\title{
Spatial modifications of three-dimensional elliptic Gaussian beam scattered by two- dimensional periodic array.
}

\author{
Alexander Gribovsky ${ }^{1}$, Oleg Yeliseyev ${ }^{1 *}$ \\ ${ }^{1}$ Institute of Radio Astronomy of NASU, Kharkov, Ukraine \\ *corresponding author, E-mail: elis seev2000@mail.ru
}

\begin{abstract}
The diffraction problem of a three-dimensional elliptic ppolarized Gaussian beam on an aperture array of rectangular holes is solved. The new algorithm for the solution of threedimensional scattering problems of linearly polarized wave beams on two-dimensional periodic structures is offered. The given algorithm allows exploring of wave beams with any allocation of a field on cross section. The case of oblique incidence of linearly polarized elliptic Gaussian wave beam on two-dimensional periodic structure is viewed. As structure the rectangular waveguides phased antenna array is chosen. The elliptic shape of a beam cross section gives the chance to proportion energy of an incident field in a plane of an antenna array in the chosen direction. The frequency dependence of the reflection coefficient intensity for the Gaussian beam is calculated. For the analysis of patterns of the reflected and transmitted beams in a far zone the frequencies on which the strongest interaction between next waveguides channels is observed have been chosen. Dynamics of patterns transformation of the reflected and transmitted beams depending on the form of cross-section and a polarization direction of an incident beam on different frequencies is investigated. It is determined that shape of the pattern of reflected and transmitted beams (symmetry, asymmetry, bifurcation, amplitude, width) depends on chosen spatial orientation of the ellipse axes of the cross section in the incident beam. Frequency ranges, in which the form of the reflected and transmitted beams is not Gaussian, are defined. The nature of transformation of the patterns of scattered beams was examined. Narrowing effect of the pattern of transmitted beam and deformation of the pattern of reflected beam is detected. A physical explanation of these effects is given. The results are presented in the form of two- and threedimensional patterns of the scattered field of beams in the far field.
\end{abstract}

\section{Introduction}

Of great interest is the investigation of electrodynamic characteristics of the reflected and transmitted elliptic beams in case of oblique incidence on semi-transparent two-dimensional periodic structures. The form of the pattern, amplitude and phase distribution of the transmitted and reflected elliptic beam fields may differ significantly from the corresponding characteristics of the fields in case of circular cross section beam scattering. To date, there are a large number of publications in which the peculiarities of scattering of two-and three-dimensional beams on different types of structures [1]-[14] are studied. In them the homogeneous dielectric slabs, one-dimensional periodic structures, two-dimensional periodic arrays of magnetodielectric layers are considered. In [15] the results of studies of electrodynamic characteristics of the Gaussian circular cross section wave beam scattering in case of normal incidence on a two-dimensional periodic structure and the calculation algorithm are presented. Oblique incidence of s-polarized beam was considered in the paper [17]. The linearly polarized beams of p-polarization, or beams with a combination of both polarizations, in which the electric field vector of each component has a nonzero pand s-projections, are widely used in practice. Herewith, the displacement and transformation of patterns shape in the scattering of beams on two-dimensional periodic structures are significantly different for $\mathrm{p}$ - and s-polarizations. It is connected with the different behavior of the scattering coefficients frequency responses for each of the plane-wave component beams of these polarizations. Also, it should be considered that in the process of reflection of beams with $p$ and s-projections of the electric field vector, intensity of scomponent is always higher.

The need for a comparative analysis of the scattering characteristics of $\mathrm{p}$-and s-polarized beams is related primarily to the fact that the beams with arbitrary polarization are formed from the $\mathrm{p}$-and s-polarized components and their different transformation upon reflection should lead to additional effects that may not have a separate place for each polarization component of the beam.

\section{Problem formulation}

Let a linearly polarized wave beam propagate from the $z>0$ half-space and be incident at an arbitrary angle on a double-periodic structure placed in the $x O y$ plane. In the general case, the unit cells of the double-periodic structure are centered at the nodes of the oblique-angled mesh lying in the $x O y$ plane and given by the angle $\chi$ between the $O x$ axis and the straight line connecting the origin and the unit cell center. The beam polarization direction is also arbitrary. It is required to find the electromagnetic field scattered by a 
double-periodic structure into free space. Fig.1 depicts the wave beam incidence and the double-periodic structure. Fig. 2 defines the $x y z$ coordinate system related to the array and the $x_{p} y_{p} z_{p}$ coordinate system related to the beam. The incident beam is given by angles $\vartheta_{0}, \varphi_{0}$ in the system $x y z$ and the coordinates $x_{0}, y_{0}, z_{0}$ locate the origin of the $x_{p} y_{p} z_{p}$ system in the $x y z$ system. Let the transverse component distribution of an incident-beam electric field in the $z_{p}=0$ plane be given as

$\vec{E}_{t}^{i}\left(x_{p}, y_{p}, 0\right)=\frac{4 \pi}{\sqrt{S}} \exp \left\{-\left(\frac{x_{p}}{w_{1}}\right)^{2}-\left(\frac{y_{p}}{w_{2}}\right)^{2}\right\}$
$\times\left(\vec{e}_{x p} \cos \alpha_{0}-\vec{e}_{y p} \sin \alpha_{0}\right)$,

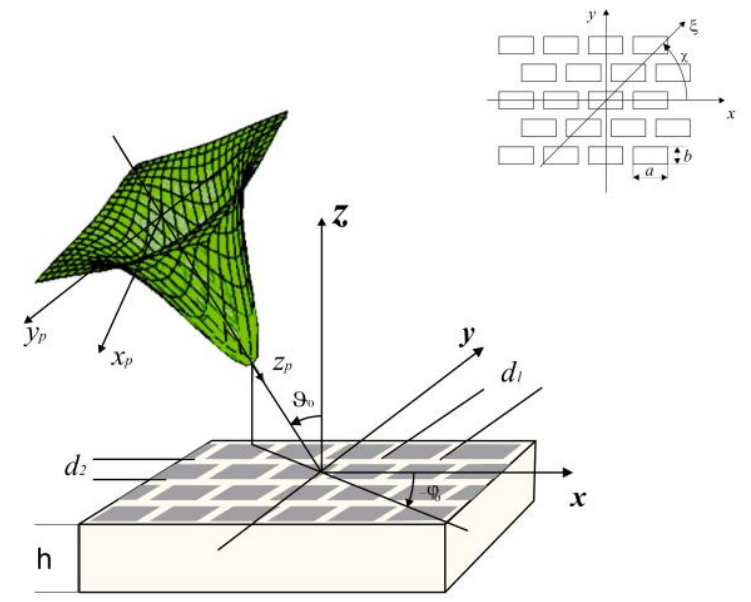

Figure 1: Gaussian beam incidence on a double-periodic structure.

where $\mathrm{S}$ is the area of the unit cell of a double-periodic structure, $w_{1}, w_{2}$ are the parameters of beam effective dimensions in the $z_{p}=0$ plane, and $\vec{e}_{x p}, \vec{e}_{y p}$ are the unit vectors of the $x_{p} y_{p} z_{p}$ coordinate system. Polarization angle $\alpha_{0}$ is defined in the $x_{p} y_{p} z_{p}$ system related to the beam (see Fig. 2). Subscript t in (1) and everywhere below stands to show that it is the transverse component of the electric or magnetic field that is considered.

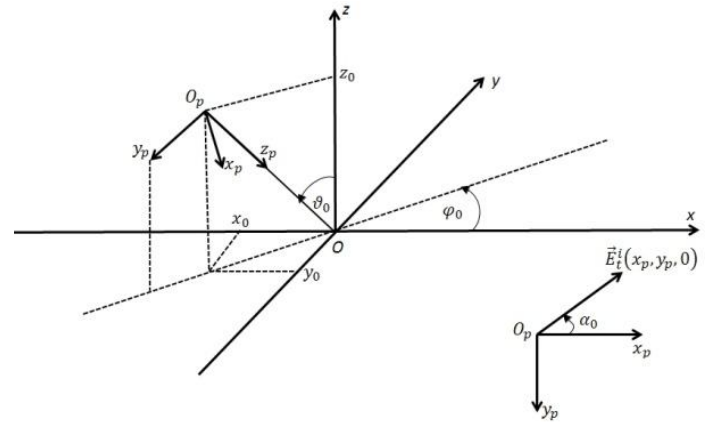

Figure 2: The coordinate system.

By the coordinate system transformation formulas, the beam electric field component transverse to the $O z$-axis in the $z=z_{p}$ plane in the $x, y, z$ coordinates is
$\vec{E}_{t}^{i}\left(x, y, z_{0}\right)=F(x, y)\left(P_{x}^{0} \vec{e}_{x}+P_{y}^{0} \vec{e}_{y}\right)$

where $F(x, y)=\frac{1}{\pi \sqrt{S}} \exp \left\{-\left(x-x_{0}\right)^{2} \delta_{1}-\left(y-y_{0}\right)^{2} \delta_{2}+\right.$ $\left(x-x_{0}\right)\left(y-y_{0}\right) \delta_{3}+i k \sin \vartheta_{0}\left[\left(x-x_{0}\right) \cos \varphi_{0}+\right.$ $\left.\left.\left(y-y_{0}\right) \sin \varphi_{0}\right]\right\}, \delta_{1}=\frac{\sin ^{2} \varphi_{0}}{w_{1}^{2}}+\frac{\cos ^{2} \vartheta_{0} \cos \varphi_{0}}{w_{2}^{2}}, \delta_{2}=\frac{\cos ^{2} \varphi_{0}}{w_{1}^{2}}+$ $\frac{\cos ^{2} \vartheta_{0} \sin ^{2} \varphi_{0}}{w_{2}^{2}}, \delta_{3}=\sin 2 \varphi_{0}\left(\frac{1}{w_{1}^{2}}-\frac{\cos ^{2} \vartheta_{0}}{w_{2}^{2}}\right)$,

$P_{x}^{0}=\sin \alpha_{0} \cos \vartheta_{0} \cos \varphi_{0}, P_{y}^{0}=\sin \alpha_{0} \cos \vartheta_{0} \sin \varphi_{0} . \quad$ The transverse component of the electric field of the incident beam is represented as the sum of the two beams with different polarizations (TE and TM polarized beams). Each of these beams can be represented as an expansion in the form of Fourier integral related to the plane TE and TM polarized waves, respectively:

$\vec{E}_{t}^{i}(x, y, z)=$
$=\frac{1}{\sqrt{S}} \int_{-\infty}^{\infty} \int_{-\infty}^{\infty} G_{1}(\xi, \zeta) \frac{\xi \vec{e}_{x}-\zeta \vec{e}_{y}}{\sqrt{\xi^{2}+\zeta^{2}}} e^{i k(x \zeta+y \xi-z \gamma)} d \xi d \zeta$
$+\frac{1}{\sqrt{S}} \int_{-\infty}^{\infty} \int_{-\infty}^{\infty} G_{2}(\xi, \zeta) \frac{\zeta \vec{e}_{x}+\xi \vec{e}_{y}}{\sqrt{\xi^{2}+\zeta^{2}}} e^{i k(x \zeta+y \xi-z \gamma)} d \xi d \zeta$

where $G_{1,2}(\xi, \zeta)$ are the incident beam spectral functions; $k=2 \pi / \lambda, \gamma=\sqrt{1-\xi^{2}-\zeta^{2}}$. The integration variables $\xi, \zeta$ have the following meanings: $\xi=\sin \vartheta \cos \varphi, \zeta=$ $\sin \vartheta \sin \varphi$, where $\vartheta, \varphi$ are the angles of incidence of a separate spatial TE or TM polarized harmonics with the amplitude $G_{1}(\xi, \zeta)$ and $G_{2}(\xi, \zeta)$, respectively. The angles $\vartheta$, $\varphi$ are determined similarly as the incidence angles $\vartheta_{0}, \varphi_{0}$ in the range of their real values.

Transverse electric components of the reflected field can be also represented as the sum of the transverse field components of two TE and TM polarized beams. Each of them is expanded in the form of Fourier integrals related to the TE and TM polarized plane waves:

$\vec{E}_{t}^{r}(x, y, z)=$
$=\frac{1}{\sqrt{S}} \int_{-\infty}^{\infty} \int_{-\infty}^{\infty} R_{1}(\xi, \zeta) \frac{\xi \vec{e}_{x}-\zeta \vec{e}_{y}}{\sqrt{\xi^{2}+\zeta^{2}}} e^{i k(x \zeta+y \xi+z \gamma)} d \xi d \zeta$
$+\frac{1}{\sqrt{S}} \int_{-\infty}^{\infty} \int_{-\infty}^{\infty} R_{2}(\xi, \zeta) \frac{\zeta \vec{e}_{x}+\xi \vec{e}_{y}}{\sqrt{\xi^{2}+\zeta^{2}}} e^{i k(x \zeta+y \xi+z \gamma)} d \xi d \zeta$

where $R_{1}(\xi, \zeta)$ and $R_{2}(\xi, \zeta)$ are unknown spectral functions, and indexes 1 and 2 correspond to the TE and TM beams, respectively.

A way of solving this problem is described in our previous paper [15] in detail. It consists in finding a relation between the unknown spectral functions $R_{1}(\xi, \zeta), R_{2}(\xi, \zeta)$ and the certain elements of generalized scattering matrix 
and the known spectral features $G_{1}(\xi, \zeta), G_{2}(\xi, \zeta)$. The given relation looks like:

$$
\begin{aligned}
& R_{1}(\xi, \zeta)= \\
& =\sum_{q=-\infty}^{\infty} \sum_{S=-\infty}^{\infty}\left\{\begin{array}{c}
G_{1}(\hat{\xi}, \hat{\zeta})_{T E} r_{q S}^{(1)}(\hat{\xi}, \hat{\zeta}) \\
\left.\quad+G_{2}(\hat{\xi}, \hat{\zeta})_{T M} r_{q S}^{(1)}(\hat{\xi}, \hat{\zeta})\right\}
\end{array}\right. \\
& \begin{array}{r}
R_{2}(\xi, \zeta)=\quad \infty \\
=\sum_{q=-\infty}^{\infty} \sum_{S=-\infty}^{\infty}\left\{G_{1}(\hat{\xi}, \hat{\zeta})_{T E} r_{q S}^{(2)}(\hat{\xi}, \hat{\zeta})\right. \\
\left.\quad+G_{2}(\hat{\xi}, \hat{\zeta})_{T M} r_{q S}^{(2)}(\hat{\xi}, \hat{\zeta})\right\}
\end{array}
\end{aligned}
$$

where $\hat{\xi}=\xi+\frac{s}{k_{2}}-\frac{q \cot (\chi)}{k_{1}}, \hat{\zeta}=\zeta+\frac{q}{k_{1}}, r_{q s}^{(1,2)}$ are the certain elements of the generalized scattering matrix of the two-dimensional periodical structure. The latter ones are found via solution of the key diffraction problem related to the spectra of the TE and TM linearly polarized plane electromagnetic waves. The upper indexes 1 and 2 correspond to the TE and TM waves, respectively. The expressions for the patterns across the field and the intensity of the reflected beam in the far-field region are obtained as follows [15]:

$D_{n \varphi}=\left|R_{1}(\vartheta, \varphi)\right| \cos \vartheta, D_{n \vartheta}=\left|R_{2}(\vartheta, \varphi)\right|$,

$D=\left(D_{n \varphi}\right)^{2}+\left(D_{n \vartheta}\right)^{2}$.

A mention should be made that the beam and array parameters can be so chosen that it would suffice to take only one series term in matrices (5). This approximation is reasoned by that the absolute value of functions $G_{1}(\hat{\xi}, \hat{\zeta})$ and $G_{2}(\hat{\xi}, \hat{\zeta})$ of the Gaussian beams is other than zero only within a small interval of angles $\vartheta, \varphi$ provided that a single spatial Floquet harmonic propagates in free space $(q=s=0)$. For $q \neq 0, s \neq 0$, the absolute values of the spectral functions tend to zero, $\left|G_{1}(\hat{\xi}, \hat{\zeta})\right| \rightarrow 0,\left|G_{2}(\hat{\xi}, \hat{\zeta})\right| \rightarrow$ 0 , in their argument domains. Then the spectral functions of the scattered wave beam come from the approximate formulas, viz.

$R_{1}(\vartheta, \varphi) \approx$

$G_{1}(\vartheta, \varphi)_{T E} r_{00}^{(1)}(\vartheta, \varphi)+G_{2}(\vartheta, \varphi)_{T M} r_{00}^{(1)}(\vartheta, \varphi)$,

$R_{2}(\vartheta, \varphi) \approx$

$G_{1}(\vartheta, \varphi)_{T E} r_{00}^{(2)}(\vartheta, \varphi)+G_{2}(\vartheta, \varphi)_{T M} r_{00}^{(2)}(\vartheta, \varphi)$,

where ${ }_{T E} r_{00}^{(1)}(\vartheta, \varphi),{ }_{T M} r_{00}^{(1)}(\vartheta, \varphi),{ }_{T E} r_{00}^{(2)}(\vartheta, \varphi),{ }_{T M} r_{00}^{(2)}(\vartheta, \varphi)$ are the amplitudes of the zeroth spatial Floquet harmonics from the solutions of the key problems of the plane linearlypolarized TE-and TM-wave diffraction by a double-periodic structure. When the array period and the wavelength are such that there are several Floquet harmonics (high-order diffraction rays) propagating in the free space, rigorous formulas (5) are used.

\section{Numerical results}

By the developed algorithm, a comparison was made between the frequency dependences of the power reflection coefficient $|R|^{2}$ of an elliptic beam and a plane wave both obliquely incident $\left(\vartheta_{0}=20 \mathrm{deg}\right)$ on a double-periodic, rectangular-meshed structure $(\chi=90 \mathrm{deg})$. The latter represents a plane perfectly-conducting screen of finite thickness $\mathrm{h}$ cross-perforated by rectangular holes. The $(a \times b)$ holes are considered as rectangular waveguides segments. Fig. 3 presents the computation results for the parameters $a=5 \mathrm{~mm}, b=1 \mathrm{~mm}, \mathrm{~h}=9 \mathrm{~mm}, d_{1}=d_{2}=$ $6 \mathrm{~mm}, \varphi_{0}=\alpha_{0}=90 \mathrm{deg}$ and $\vartheta_{0}=20 \mathrm{deg}, w_{1}=30 \mathrm{~mm}$, $w_{2}=10 \mathrm{~mm}$. The Figure shows that in the frequency band under consideration, the frequency dependences of the power reflection coefficients of the incident wave beam and the incident plane wave are practically coinciding. The only exceptions are the frequencies points $(f=37.2 \mathrm{GHz}$ and $f=36.8 \mathrm{GHz}$ ), where the frequency is in proximity of the "sliding" point, where the high-order spatial Floquet harmonics start traveling. In this case, the spatial distributions of the scattered electromagnetic fields of the plane wave and the wave beam are most different. Power reflection coefficient is calculated for the transverse electric field component, excluding the longitudinal component, which, at oblique incidence and p-polarization, is different from zero. Therefore, in case of total reflection, the maximum value of the reflection coefficient of the power is less than one. The patterns across the intensity $D$ were calculated for the incident, transmitted and reflected beams at the oblique beam incidence on the structure. The patterns were evaluated in the proximity of the frequency $f=37.2$ $\mathrm{GHz}$ corresponding to the second extremum of the functions from Fig.3. Note that the largest changes in pattern shapes for the beam and screen parameters as chosen are observed in the plane approaching $\varphi=90 \mathrm{deg}$. Fig. 4(a) and 4(b) plots the calculated fixed-frequency field patterns for an elliptical-section beam obliquely incident on an aperture array with a right-angled mesh $(\chi=90 \mathrm{deg})$ in the plane $\varphi=90 \mathrm{deg}$. The screen and beam parameters are as right before.

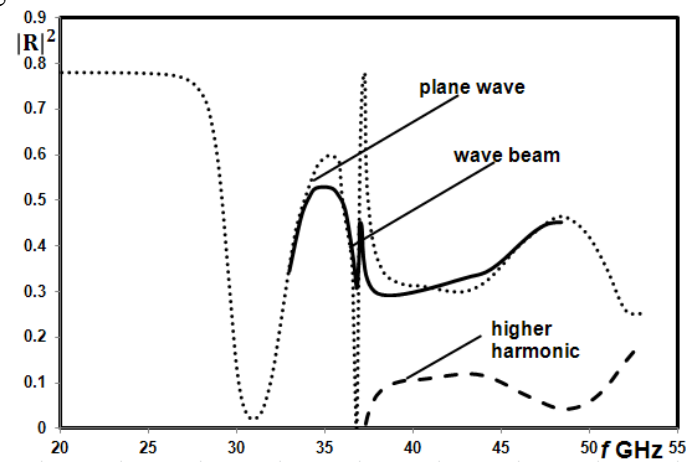

Figure 3: The comparison of the frequency dependences of the reflection coefficient intensity for the plane wave and Gaussian beam.

From Figures it is seen that the patterns of the reflected and transmitted beams, unlike the incident beam pattern, change their shapes in the $\varphi=90 \mathrm{deg}$ plane. Narrowing and 
maxima shift of the transmitted field pattern occurs. The reflected field patterns are distorted. The narrowing effect of the transmitted field pattern has been already observed [16] when characteristics of the two-dimensional Gaussian beam scattering from a single-periodic strip array were studied. The effects of narrowing the pattern of the scattered field and the shift of the maximum of pattern of the transmitted field, in the case of oblique incidence of spolarized elliptical beam, are described and explained in the paper [17].

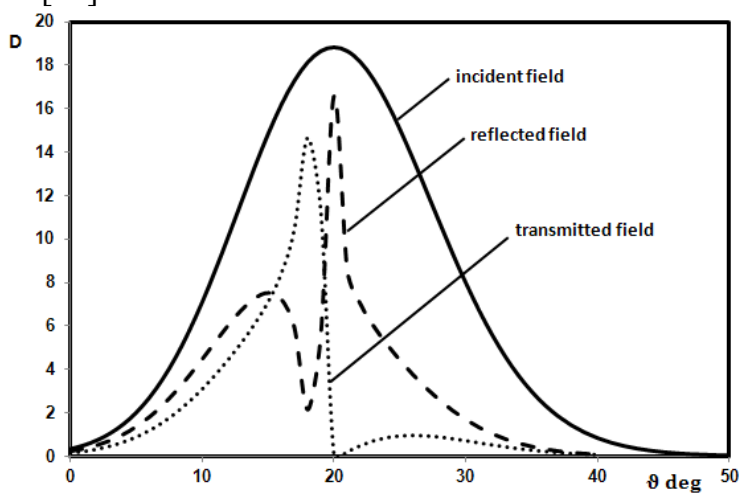

Figure: 4a. The field pattern. $f=37.2 \mathrm{GHz}, w_{1}=$ $30 \mathrm{~mm}, w_{2}=10 \mathrm{~mm}$.

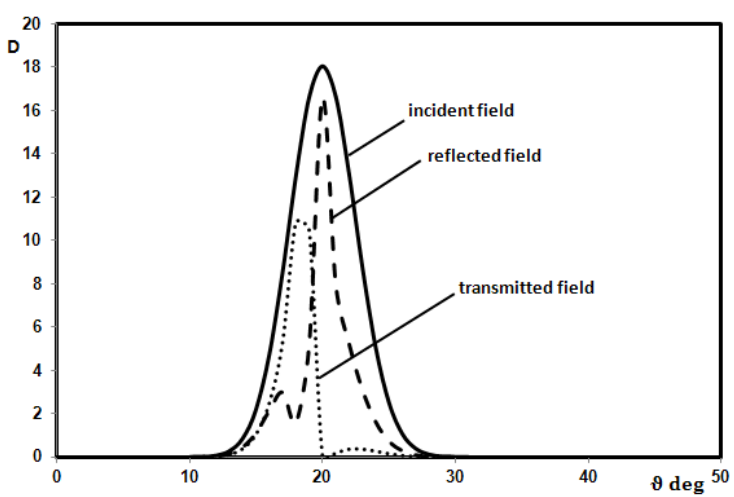

Figure: $4 \mathrm{~b}$. The field pattern. $f=37.2 \mathrm{GHz}, w_{1}=$ $10 \mathrm{~mm}, w_{2}=30 \mathrm{~mm}$.

For clearness sake the tree-dimensional patterns across the intensity are evaluated. Fig. 5(a) and Fig. 5(b) present the incident field patterns of the elliptic beam in Cartesian and 3-D polar coordinate system Three-dimensional patterns of power in the far zone for the reflected and transmitted fields were calculated at a frequency $f=37.2 \mathrm{GHz}$. Fig. 6 and Fig. 7 show the patterns of the scattered field for two cases of spatial orientation of the ellipse of cross-section of the incident beams. In the case when $w_{1}>w_{2}$, the semimajor axis of the ellipse of cross-section of the beam is directed along the axis $O x$, and in the case when $w_{1}<w_{2}$ - along the axis $O y$. Comparative analysis of patterns in the far field for the cases of scattering of s- and p-polarized beams has shown that restriction of patterns, as the transmitted and reflected fields, compared with the pattern of the incident field is also observed for p-polarization. In addition, the shift of the maximum of the pattern of the transmitted field also occurs, compared to the position of the maximum in the pattern of the incident field. However, there is a significant difference in the patterns shapes of the scattered field.
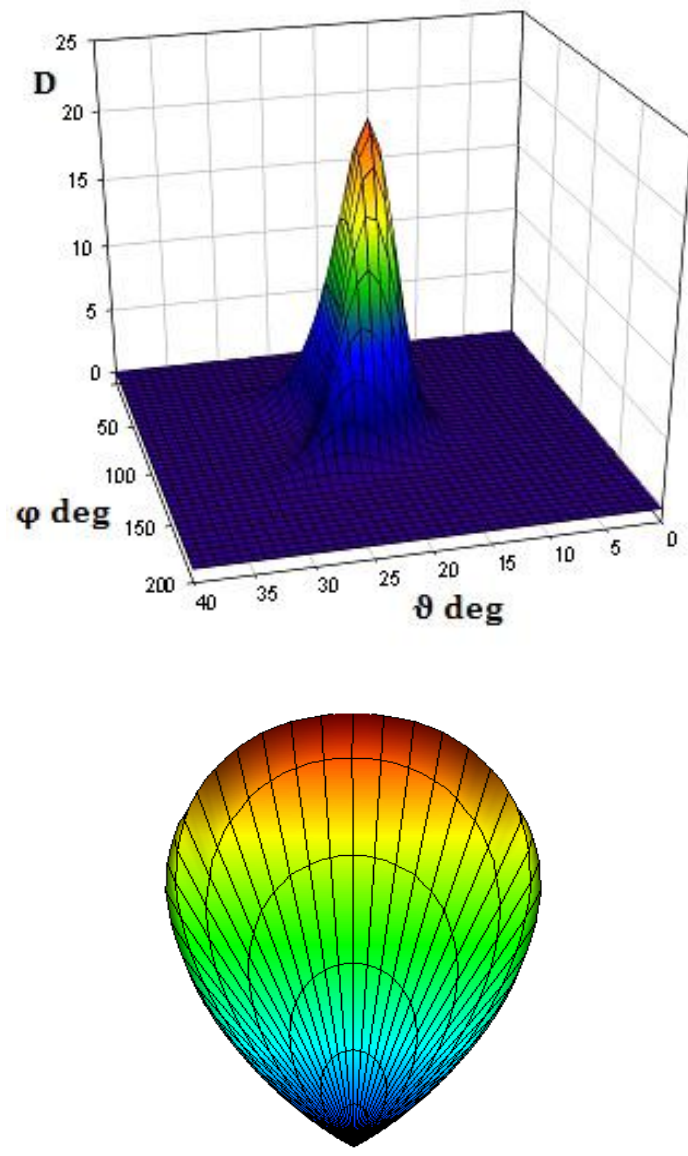

Figure 5a: The incident field pattern $w_{1}=10 \mathrm{~mm}, w_{2}=$ $30 \mathrm{~mm}$.

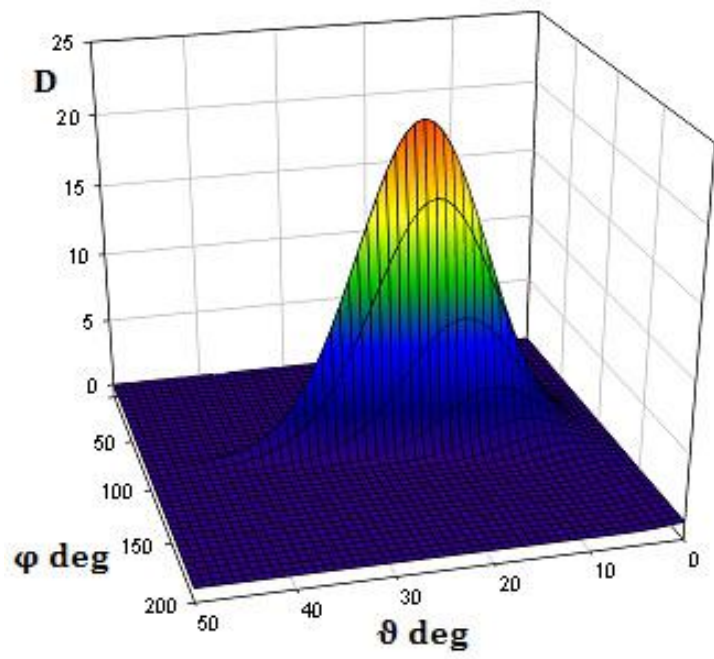




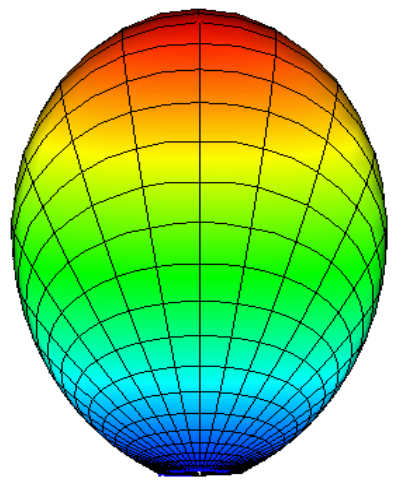

Figure 5b: The incident field pattern $w_{1}=30 \mathrm{~mm}, w_{2}=$ $10 \mathrm{~mm}$.

Numerical studies have shown that a direction of orientation in space of the axes of ellipse of cross-section beams has a significant influence on the shape of the patterns of the scattered field in the case of p-polarization besides the polarization direction of obliquely incident beams. Thus, at oblique incidence of p-polarized beam, a large axis of the ellipse which is directed along the axis $O y$, the patterns of the scattered field have multi-lobe nature (see Fig. 7), which was not observed in the case of s-polarization. This is explained by the difference in amplitude and phase distributions of the electromagnetic field in the plane of the array for these polarizations, different conditions of excitation of the waveguide waves in the waveguide channels, as well as the interference of the field reflected from the plane of the array aperture and the field radiated from the waveguide channels.

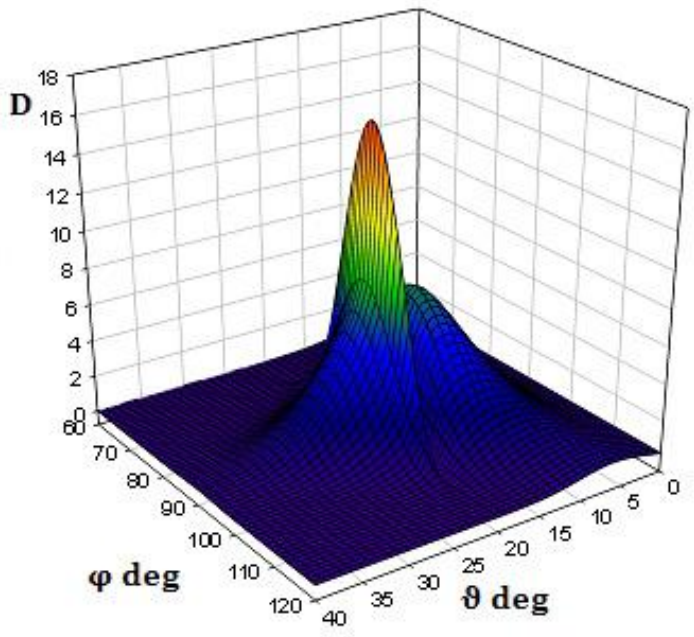

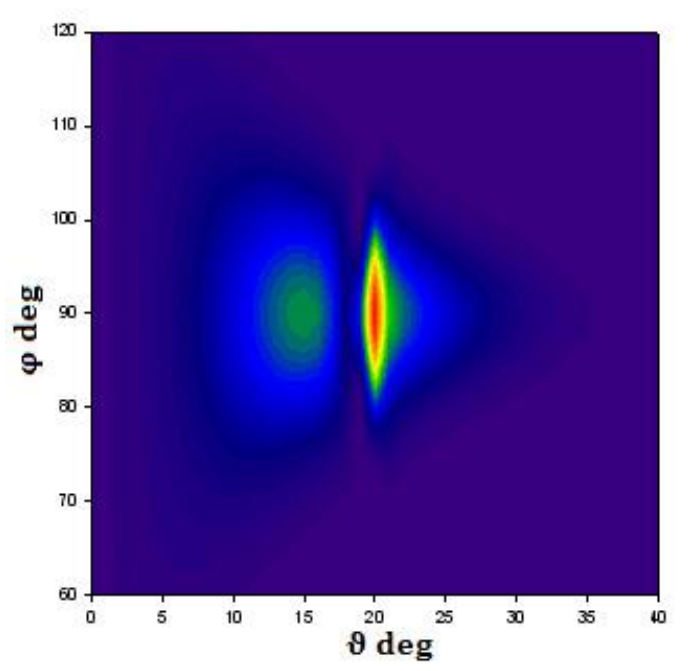

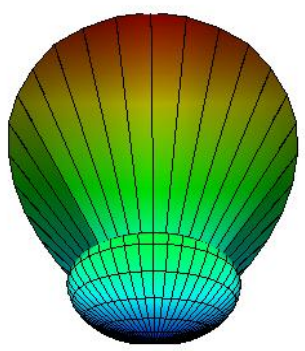

Figure 6a: The pattern of reflected field $f=37,2 \mathrm{GHz}$ $w_{1}=30 \mathrm{~mm}, w_{2}=10 \mathrm{~mm}$.

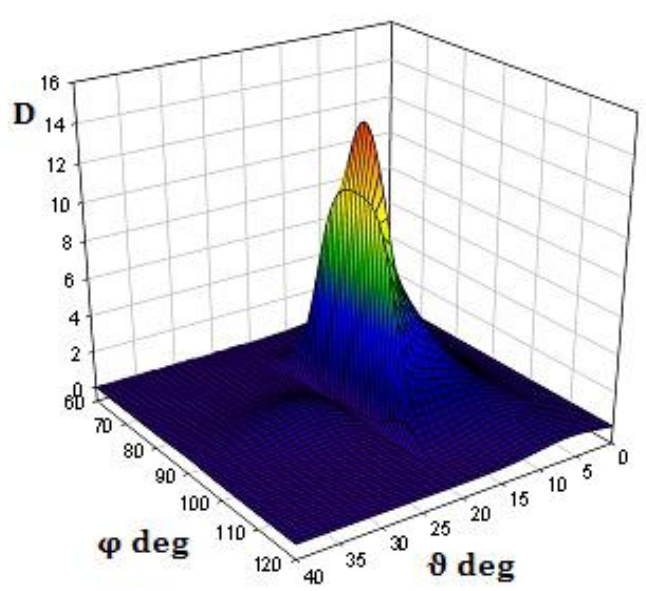



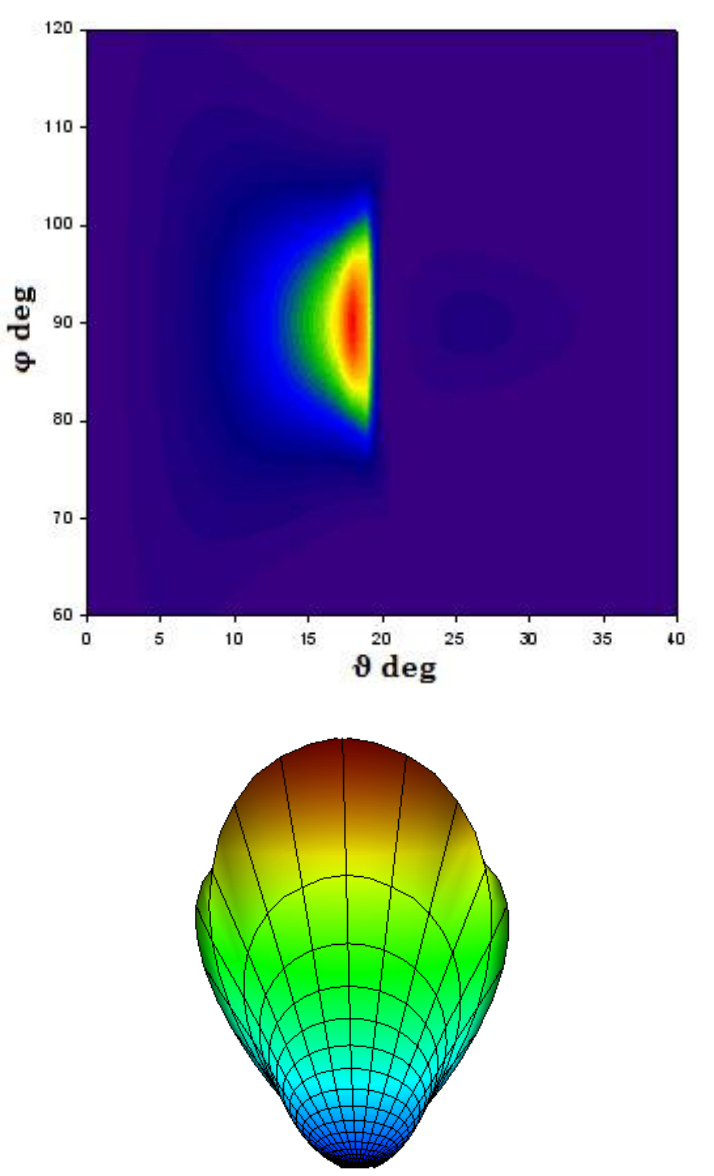

Figure 6b: The pattern of transmitted field $f=37,2 \mathrm{GHz}$ $w_{1}=30 \mathrm{~mm}, w_{2}=10 \mathrm{~mm}$.

The method of forming multi-lobe patterns in phased arrays of rectangular waveguide apertures by excitation of the array with the obliquely incident p-polarized Gaussian wave beam with an elliptical cross-sectional shape, without any additional devices, may be of great practical importance. Numerical studies have shown that the lobe amplitude and their number depend on the parameters of the incident beam, at constant geometric parameters of the phased array.

The studies conducted in this work and the results published in the paper [17], where the case of s-polarization is examined, allow drawing general conclusions. The effects of the restriction of the patterns of scattered field, the shift of the maximum in the pattern of transmitted field are observed at frequencies near the "sliding" points. In these cases, the amplitude of surface waves propagating along the aperture of phased array, sharply increase and the degree of interaction between adjacent waveguide channels rises. This also increases the effective radiating surface of the antenna array, compared with the surface, which is "highlighted" with the wave beam.
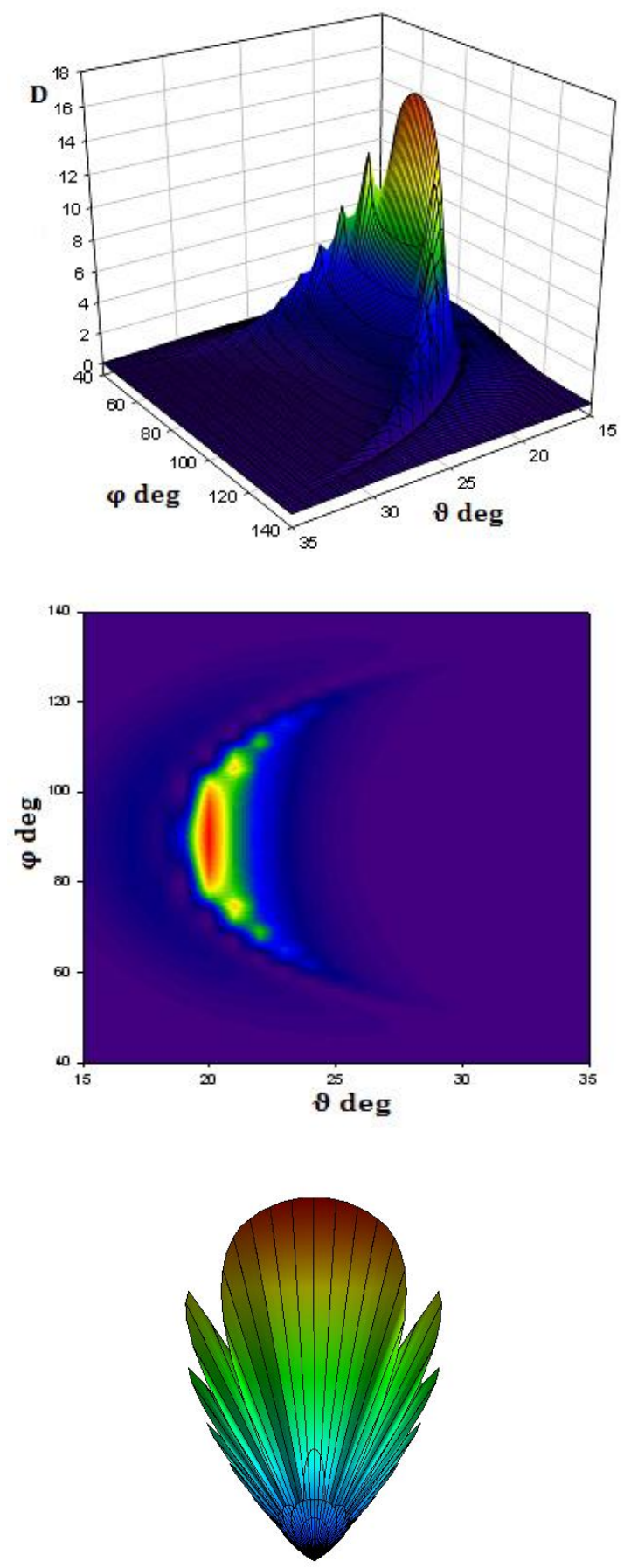

Figure 7a: The pattern of reflected field $f=37,2 \mathrm{GHz}$ $w_{1}=10 \mathrm{~mm}, w_{2}=30 \mathrm{~mm}$. 

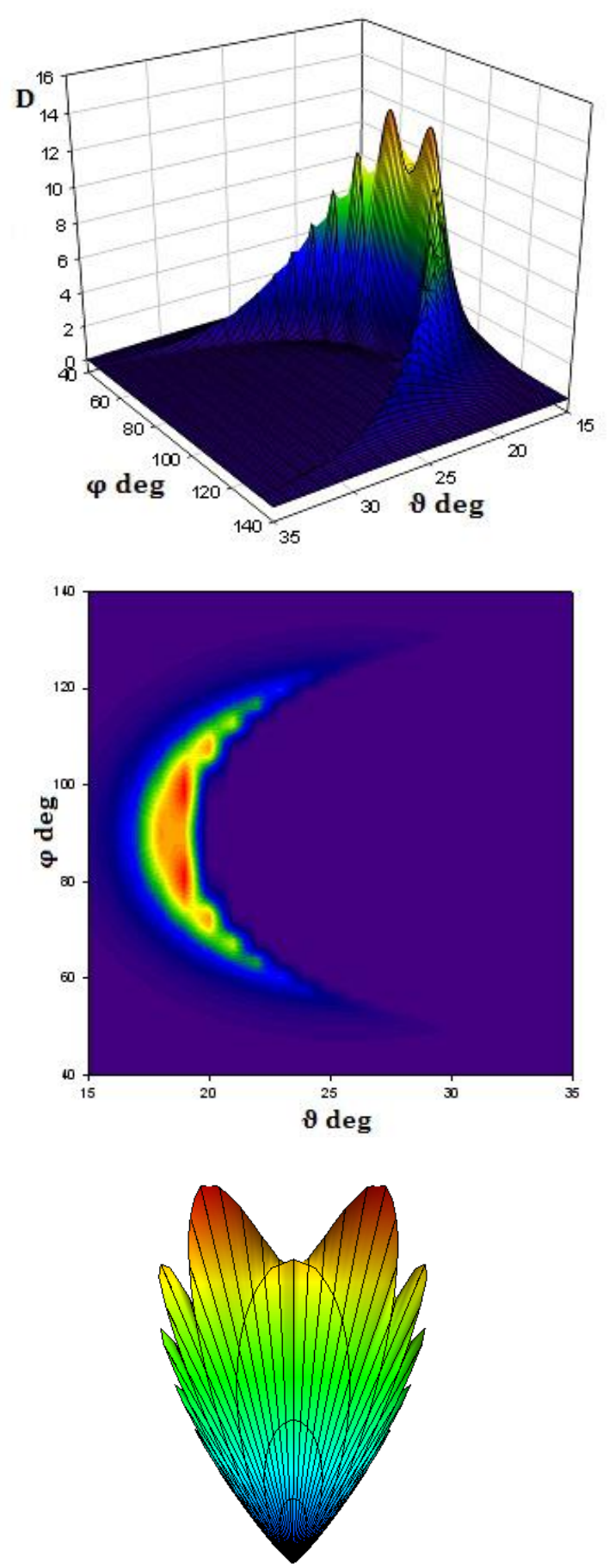

Figure 7b: The pattern of transmitted field $f=37,2 \mathrm{GHz}$ $w_{1}=10 \mathrm{~mm}, w_{2}=30 \mathrm{~mm}$.

\section{Conclusions}

In the present work, computational algorithm has been developed to obtain the electrodynamic characteristics of scattering of tree-dimensional Gaussian beams from doubleperiodic structures. For a plane wave and a wave beam, ( $\left.\alpha_{0}=\varphi_{0}=90 \mathrm{deg}\right)$ both obliquely incident on a plane finite-thick double-periodic screen perforated by rectangular holes, the frequency dependence of the power reflection coefficients were compared. The difference in the behavior of the frequency dependences of power reflection coefficients for the plane wave and the wave beam is shown. For an obliquely incident elliptic p-polarized beam, the field patterns of screen-scattered beams were calculated. The narrowing effect was observed for a pattern of the transmitted beam field, while the incident beam pattern was unchanged. It is determined that in the case of $p$ polarization the same effects of restriction of the patterns of scattered field and the shift of the maximum in the pattern of reflected field, as in the case of s-polarization, are observed. However, shapes of the patterns of scattered field in the case of oblique incidence of $\mathrm{p}$ - and s-polarized Gaussian elliptical wave beams in phased antenna array of rectangular waveguides segments are significantly different. For certain parameters of obliquely incident p-polarized wave beam, patterns of the scattered field have multi-lobe character. The developed algorithm enables the examination of electrodynamical characteristics of scattering of three-dimensional Gaussian beams from plane double-periodic structures provided that the key problem solution of plane linearly-polarized electromagnetic wave diffraction from this structure is available.

\section{References}

[1] T. Tamir, H. L. Bertoni, Lateral displacement of optical beams at multilayered and periodic structures,Jornal of the Optical Society of America, Vol. 61, No. 10, 1397 1413, October 1971 .

[2] A. V. Gribovsky, A new method of controlling the radiation in waveguide phased array, Telecommunications and Radio Engineering, Vol. 61, No. 1, 58-66, 2004.

[3] A. V. Gribovsky, S. L. Prosvirin, Scattering of a wave beam by a reflected array made of rectangular waveguides, Journal of Communications Technology and Electronics, Vol. 42, No. 9, 961-972, 1997.

[4] S. S. Tretyakova, O. A. Tretyakov, V. P. Shestopalov, Wave beam diffraction on plane periodical structures,Radiotekhnika\& Electronica, Vol. 17, 13661373, July 1972 (in Russian).

[5] S. Y. Shin, L. B. Felsen, Lateral shift of totally reflected Gaussian beams, Radio Sci. ,Vol. 12, 551564, July 1977.

[6] L. N. Litvinenko, S.L. Prosvirnin, Spectral scattering operators in problems of wave diffraction on flat screens,Nauk. Dumka, 1984.

[7] O. A. Godin, Diffraction theory of lateral shift of bounded wave beams during reflection,Zh. Tekh. Fiz., Vol. 54, No. 4, 2094-2104, 1984; Vol. 55, No. 2, 17 25, 1985 (in Russian).

[8] R. P. Riesz, R. Simon, Reflection of Gaussian beam from a dielectric slab, J. Opt. Soc. Am. A, Vol. 2, 1809-1817, 1985.

[9] J. Maciel, L. Felsen, Gaussian beam analysis of propagation from an extended plane aperture distribution through dielectric layers, IEEE Trans. 
Antennas Propagat., Vol. AP-38, 1607-1624, October 1990.

[10] S. N. Shulga, Two-dimensional wave beam scattering on an anisotropic half-space with anisotropic inclusion, Optics and Spectroscopy, Vol. 87, 503-509, March 1999 (in Russian).

[11] V. R. Tuz, Three-dimensional Gaussian beam scattering from a periodic sequence of bi-isotropic and material layers, Progress In Electromagnetics Research B, Vol. 7, 53-73, 2008.

[12] Z. Wu, L. Guo, Electromagnetic scattering from a multilayered cylinder arbitrarily located in a Gaussian beam, a new recursive algorithms, Progress In Electromagnetics Research, PIER, Vol. 18, 317-333, 1998.

[13]T. Shen, W. Dou, Z. Sun, Gaussian beam scattering from a semicircular channel in a conducting plane, Progress In Electromagnetics Research, PIER, Vol. 16, 67-85, 1997.

[14] W. T. Dong, L. Gao, C. W. Qiu, Goos-Hänchen shift at the surface of chiral negative refractive media, Progress In Electromagnetics Research, PIER, Vol. 90, 255-268, 2009.

[15]A. V. Gribovsky, O.A. Yeliseyev, Gaussian beam scattering on two-dimensional periodic aperture array, Progress In Electromagnetics Research B, Vol. 31, 323337, 2011.

[16] A. V.Gribovsky, S. L. Prosvirin, I. I. Reznik, Reflective phased array antenna of rectangular waveguides of finite depth, Radio Physics and Radio Astronomy, Vol. 2, No.1, 52-60, 1997 (in Russian).

[17]A. V.Gribovsky and O.A. Yeliseyev, The elliptic Gaussian beam scattering on phased antenna array with rectangular waveguides, Progress In Electromagnetics Research M, Vol. 22, 109-121, 2012. 